\title{
Visual perception of 3D printed translucent objects
}

\author{
Bui Minh Vu ${ }^{1}$, Philipp Urban ${ }^{2}$, Tejas Madan Tanksale ${ }^{2}$, Shigeki Nakauchi ${ }^{1}$ \\ ${ }^{1}$ Toyohashi University of Technology, Japan. \\ ${ }^{2}$ Fraunhofer Institute for Computer Graphics Research IGD, Germany.
}

\begin{abstract}
In order to reproduce translucent objects by $3 D$ printers employing fully transparent (or clear) material, modeling the human visual perception of translucency is crucial. In this preliminary study, a set of 256 texture-less samples was created by mixing white and clear materials using multi-jet $3 D$ printing. The samples differ in both lateral light transport properties and transmittance. Two psychophysical experiments were conducted to reveal the relationship between transmittance and a perceptually uniform scale for translucency. The results show that Stevens' power law describes well this relationship within the optically thin range of samples. Furthermore, the sensitivity to lateral light transport is small compared to transmittance for the texture-less sample set.
\end{abstract}

\section{Introduction}

Multi-jet or Polyjet 3D printers, such as the Stratasys Objet Connex series [1], are able to combine multiple UV curable materials on a droplet level into a single object. In contrast to $3 \mathrm{D}$ printers using powder blinder [2] and layer-laminated manufacturing [3] technologies, multi-jet 3D printers are capable of creating objects of spatially-varying color, translucency and haptical properties. In addition, multi-jetting allows for high resolution prints and can produce smooth surfaces, making it very suitable for appearance reproduction.
Multi-jet 3D printers have been used to create desired singletone subsurface scattering properties [4], opaque objects inside transparent shells [5], and for full color printing [6][7] .

Nevertheless, an errorless reproduction of given optical material properties - described by the spatially-varying Bidirectional Surface Scattering Reflectance Distribution Function (BSSRDF) [8], - is rather unlikely, because of limitations in absorption, scattering and refractive indices of available inks. An optimal reproduction is, therefore, a reproduction that minimizes perceptual errors for specified viewing conditions subject to device limits.

For this reason, modeling the visual perception of translucency is crucial if we aim to reproduce translucent objects by $3 \mathrm{D}$ printers. Real world materials reach from fully opaque, e.g. metals, to fully transparent, e.g. glass, and span intermediate degrees of translucency such as wax, marble, or human skin.

In this work, we focus on the visual perception of translucency for 3D printed objects. We conduct two experiments to reveal the relationship between a sample possessing a defined white-clear material ratio and a perceptually-uniform translucency scale. In the first experiment we used the Method of Constant Stimuli with probit analysis [9] and in the second experiment the Maximum Likelihood Difference Scaling method [10][11].

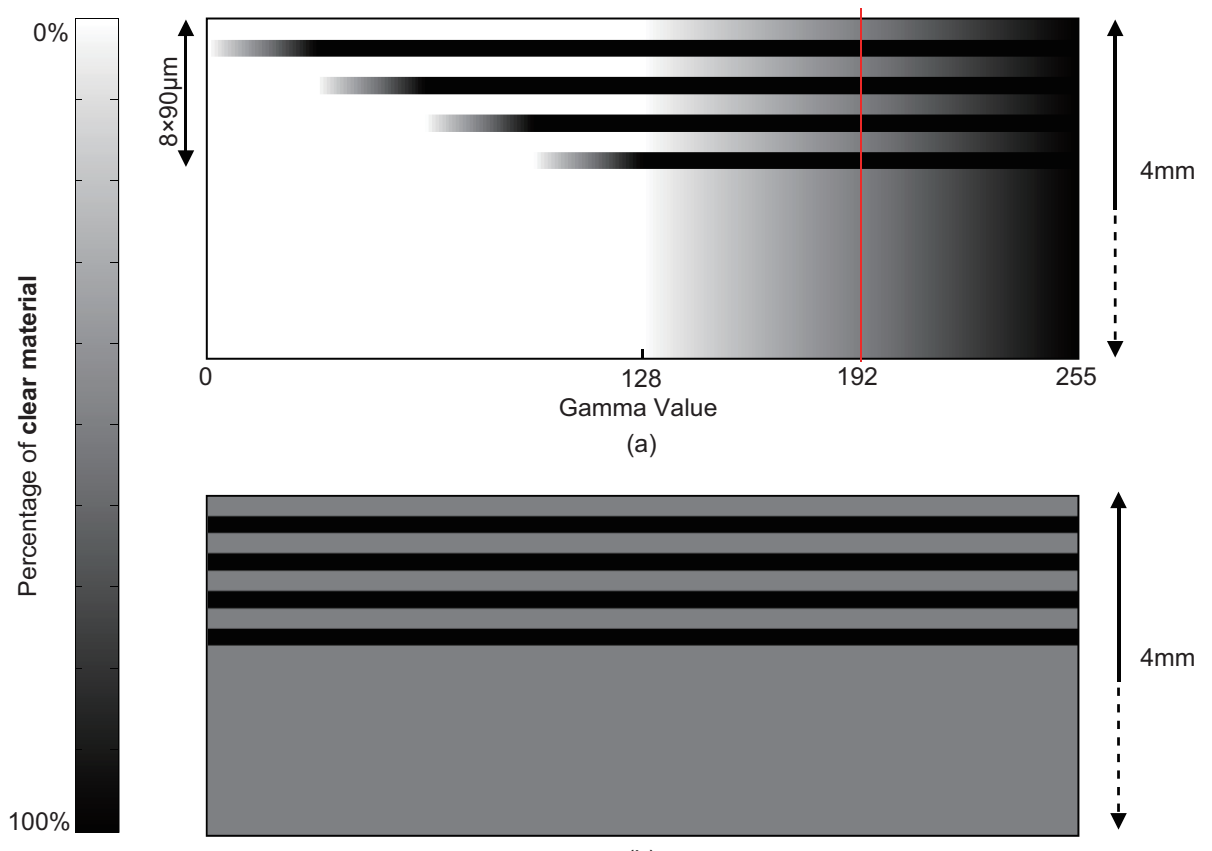

(b)

Figure 1. Sample cross section showing the distributions of clear material (a) and an example of sample with gamma value of 192 (b) 
A psychometric function is determined by translucency measurements of the samples used in the experiment, relating physical measurements to the perceptual-uniform translucency scale. Such a function is necessary for translucency gamut mapping and to obtain perceptually meaningful error rates. Both are important factors in the reproduction of translucency.

\section{Psychophysical experiment Samples}

All samples were printed using the recently introduced Stratasys $\mathbf{J} 750$ multimaterial printer employing the standard VeroPureWhite and VeroClear material. For both materials, absorption coefficients are almost similar and very small over the whole visible wavelength range. The materials mostly differ in scattering coefficients: The clear material has scattering coefficients of almost zero while the white material has much larger scattering coefficients.

An 8-bit encoded parameter called gamma value controls the amount of clear material within a sample. The sample with a gamma value of 0 contains only white material. The sample with a gamma value of 255 contains only clear material. The distribution of clear material for intermediate gamma values is shown in Figure 1. Figure 1(a) shows that for gamma values smaller than 128 , clear material is only added up to $8 \times 90 \mu \mathrm{m}=0.72 \mathrm{~mm}$ beneath the surface and that up to four layers of fully clear material are created in this distance range. In the gamma value range of 128-255 also white material is replaced by clear material deeper in the sample.

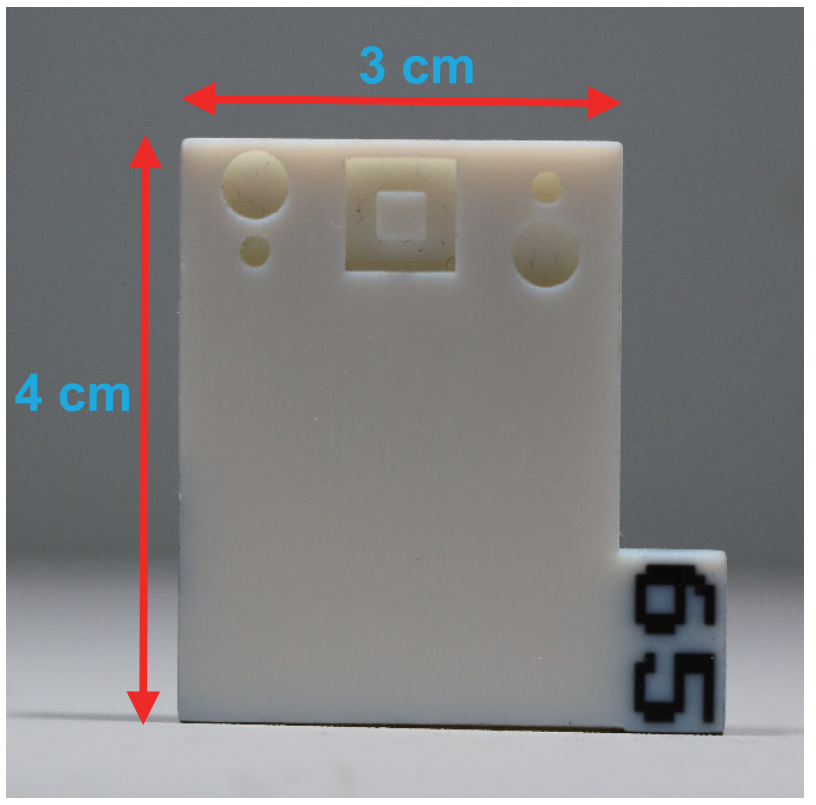

Figure 2. Sample.

Note that Figure 1(a) shows a sample with a gradient of gamma values. Such a sample was not used in this study. Each sample has only one gamma value assigned. Figure 1(a) was only included for illustration purposes because each vertical line corresponding to a particular gamma value shows the clear material distribution within the sample associated to the same gamma value. An example is shown in Figure 1(b) for a gamma value of 192 corresponding to the red line in the upper image. The aim of replacing clear materials in this way is to alter mostly lateral scattering for gamma value below 128 , because light transport within clear material layers is larger than through white material.

The sample size was $3 \mathrm{~cm} \mathrm{x} 4 \mathrm{~cm}$ with $4 \mathrm{~mm}$ of thickness which is shown in Figure 2. The samples were divided into 2 spatial parts: one part is flat, and the other has features for cues which should help observers to judge the translucency [12]. These features are holes of different diameters to create edges and shadows. The samples were randomly numbered, to avoid any indications of the gamma value used.

\section{Holder}

We used the sample holder shown in Figure 3 for our experiments. It is covered with an almost opaque matte gray coating to minimize subsurface light transport between samples and holder that might bias the translucency judgements. For the same reason, we have not placed the samples side-by-side but added a $1 \mathrm{~cm}$ gap between them.

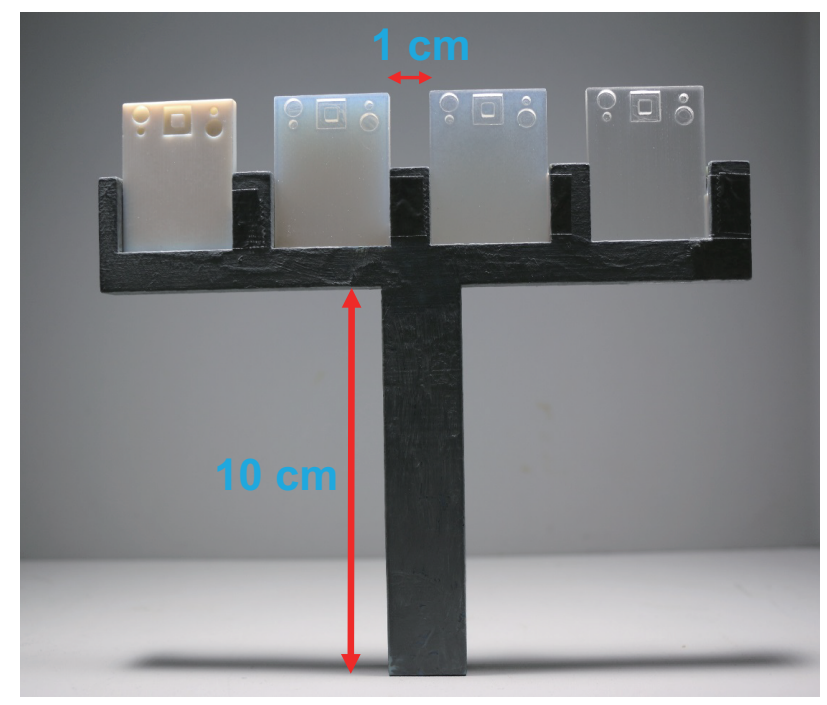

Figure 3. Holder.

\section{Method of Constant Stimuli with Probit Analysis}

This method is well known in the color science community to measure suprathreshold color differences [9][13]. A center and test sample is presented to observers juxtaposed with an anchor pair (Figure 3). The observers are asked to judge which pair has the larger translucency difference. By using test samples with varying gamma values, we can obtain gamma values around the center sample with the same perceived difference than that of the anchor pair. For this, probit analysis is used on the binary decisions given by the observers to obtain a median threshold representing the perceived translucency difference $(50 \%$ of the observers judge the center/test pair to have a smaller difference than the anchor pair and 50\% judge this difference to be greater).

The gamma values of the anchor pair and 4 center samples are shown in Table 1. The center samples were selected to cover the whole gamma range and have roughly equidistant perceived 
difference. Figure 6 shows the anchor pair which is used in the experiment. For each but the first center sample, two directions are investigated (one for bigger and one for smaller gamma values than the center sample). For the first center sample with the gamma value of 0 , only one direction is possible (bigger gamma values). In total 7 median thresholds around the considered 4 center samples are investigated. In each direction of a center sample, 6 test samples were selected ensuring that the maximum perceived difference is clearly larger than that of the anchor pair. They were presented with the center sample in random order for each observer. The randomization of the stimuli order was intended to minimize any sequential effects. A total of 42 different sample pairs were presented to each observer alongside with the anchor pair.

Tabble 1. Gamma value of selected samples in each method.

\begin{tabular}{|c|c|c|}
\hline \multicolumn{2}{|c|}{ Method } & Gamma value \\
\hline Method of & Anchor pair & 254,255 \\
\cline { 2 - 3 } Constant Stimuli & Center samples & $0,127,223,247$ \\
\hline \multicolumn{2}{|c|}{ Maximum Likelihood } & $0,63,127,191$, \\
\multicolumn{2}{|c|}{ Difference Scaling } & $223,239,247,255$ \\
\hline
\end{tabular}

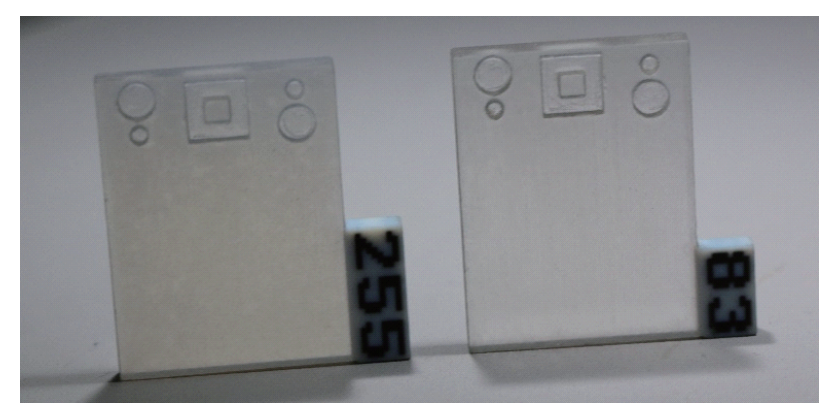

Figure 6. Anchor pair used in the Method of Constant Stimuli.

\section{Maximum Likelihood Difference Scaling method}

In this method, 2 pairs of the test samples (4 samples) are presented to observers side-by-side in the sample holder. Observers are asked to judge which pair has a bigger translucency difference.

We used 8 test samples which are shown in Figure 4. The gamma values of 8 test samples are given in Table 1 . The observers were presented all combinations of the test samples in random order. Each observer had to make a total of 70 decisions.

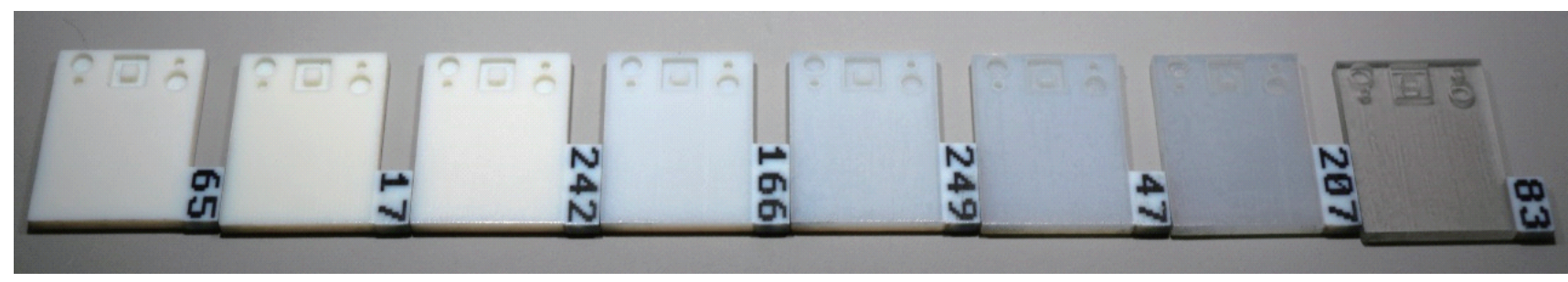

Figure 4. 8 test samples used in the Maximum Difference Scaling method.

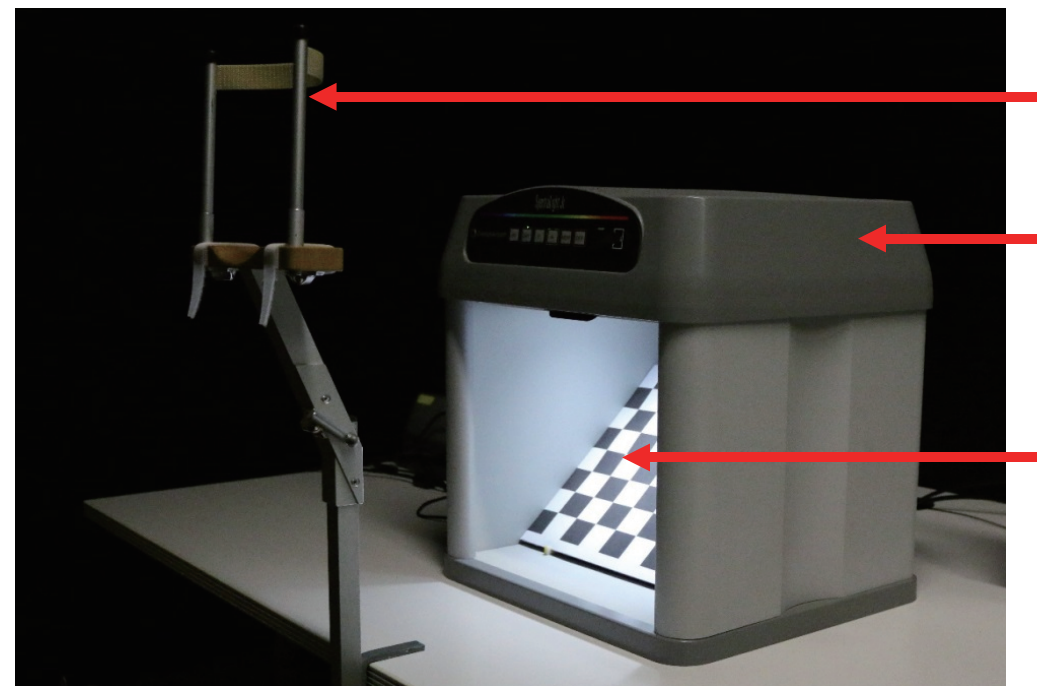

Chinrest

- $45^{\circ} / 0^{\circ}$ geometry

Viewing booth

[GretagMacbeth SpectraLight Jr.]

- $\quad$ Day light (D50)

Checker board

- Center white patch: $446 \mathrm{~cd} / \mathrm{m}^{2}$

- Center black patch: $79.73 \mathrm{~cd} / \mathrm{m}^{2}$

Figure 5. Physical setup for the experiment. 


\section{Experimental setup and procedure}

Figure 5 shows the physical setup for both psychophysical experiments. They were conducted in a dark room to minimize stray light. Samples were illuminated by the SpectraLight Jr. viewing booth (GretagMacbeth) with a filtered-tungsten day light (D50) illuminant. A checkerboard was placed into the viewing booth with a surface normal facing to the observer giving him additional cues to judge translucency. The luminance of light emitted from the center white patch of the checkerboard was 446 $\mathrm{cd} / \mathrm{m}^{2}$ and that of the center black patch was $79.73 \mathrm{~cd} / \mathrm{m}^{2}$.

The observers used a chin rest during the experiment. The distance between the chin rest and the center of the checkerboard was $50 \mathrm{~cm}$. The viewing conditions were adjusted to approx. $45^{\circ} / 0^{\circ}$ geometry (illumination/detection) for avoiding specular highlights. Observers held the holder at their arm's length during the experiment and were allowed to tilt the holder.

Prior to each experiment, observers had to look into the viewing booth for one minute to adapt to its luminance. The observers were given unlimited time to decide. There were 5-10 minutes break between the two experiments.

\section{Observers}

5 observers ( 4 males, 1 female) participated in the experiment. The age of the observers ranged from 23 to 30 years old with the average age of 26.2. All observers had normal or corrected to normal visual acuity according to the Snellen test.

\section{Results and Discussions Method of Constant Stimuli with Probit Analysis}

Results were evaluated by probit analysis for each direction around a center sample. We fitted a cumulative binomial distribution to frequencies of binary choices (test pair has larger/smaller difference than the anchor pair 1/0) using the MATLAB function glmfit. A fitting example for center sample 247 in the direction of smaller gamma values is shown in Figure 7.

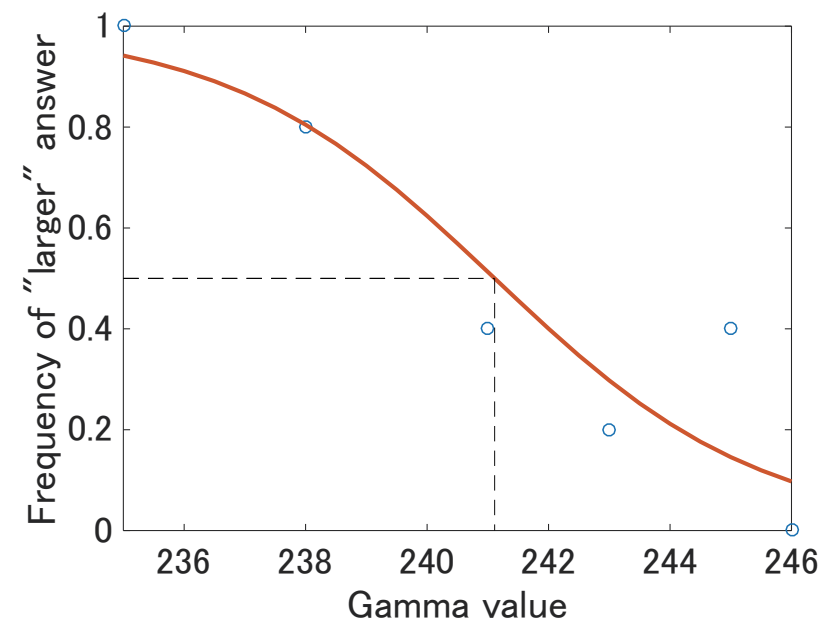

Figure 7. Fitting 1 direction of center sample with gamma value 247.

We inverted each of the 7 fitted cumulative binomial distributions at probability 0.5 to obtain the median threshold (T50). In this way, 7 gamma values were obtained and result, together with the gamma values of the corresponding center samples, in 7 gamma value differences having the same translucency difference than the anchor pair. By using these pairs, we obtained the slope of the function relating gamma values to the perceptuallyuniform translucency scale at multiple points (derivatives at multiple points) as shown in Figure 8.

We selected the power function:

$$
f(\gamma)=a \gamma^{b}
$$

to fit these points (using the Curve Fitting Toolbox from MATLAB [14]) because the fit shows the highest coefficient of determination $\left(R^{2}=0.93\right)$ in comparison with other tested functions (we tested also Exponential and Polynomial functions). The result of the fitting is also shown in Figure 8.

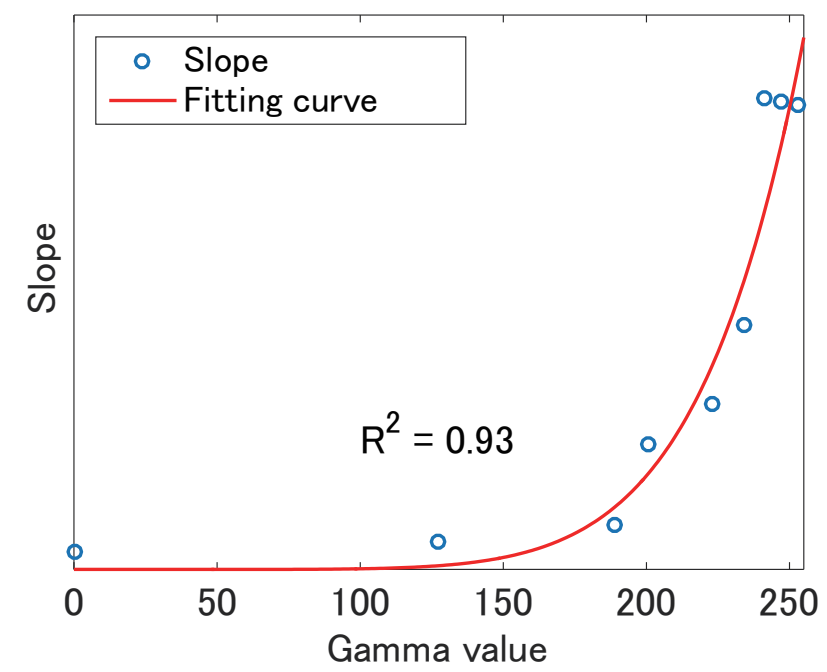

Figure 8. Slope of the psychometric function and the fitted power function.

In order to obtain the psychometric function, we take the integral of the fitted curve which is still a power function;

$$
F(\gamma)=\int f(\gamma)=\frac{a}{b+1} \gamma^{b+1}+C
$$

where $C$ is the constant of integration.

Finally, we rescaled the resulting function using;

$$
\begin{array}{r}
g(\gamma)=\frac{1}{F(255)-F(0)}[F(\gamma)-F(0)] \\
\text { where } \quad \gamma \in[0,255]
\end{array}
$$

so that $g(\gamma)$ ranges from 0 to 1 . The resulting function is shown in Figure 9(a).

\section{Maximum Likelihood Difference Scaling}

From the pattern of responses, the perceptually uniform translucency scale can be estimated. We used Palamedes Toolbox [15] to analyze the response data. The scale estimated by the Maximum Likelihood Difference Scaling method is shown in Figure 9(b). The details of how the perceptual scale is inferred from the pattern of the responses are provided in [10]. 


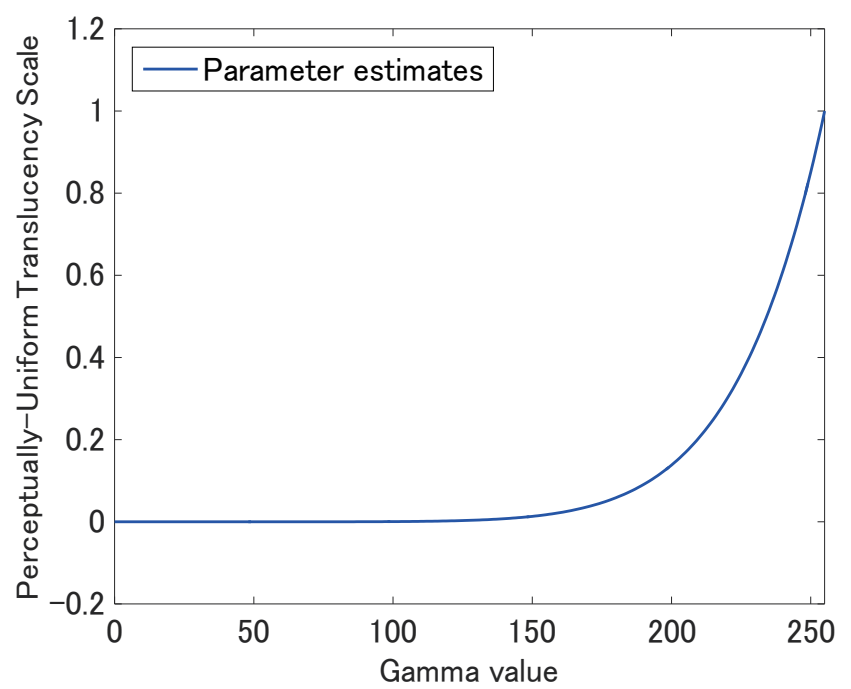

(a)

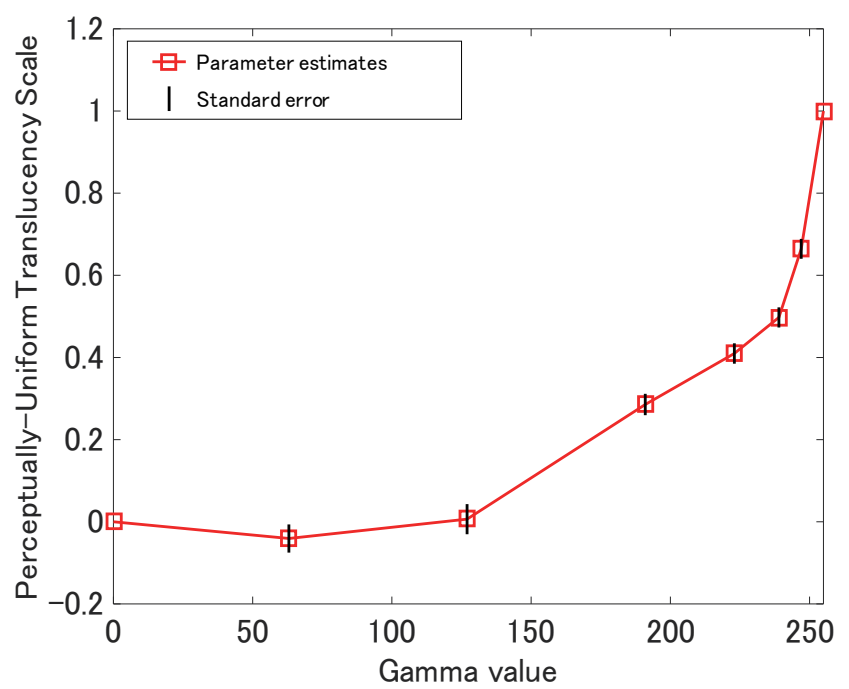

(b)

Figure 9. Results from the Method of Constant Stimuli (a) and the Maximum Likelihood Difference Scaling (b).

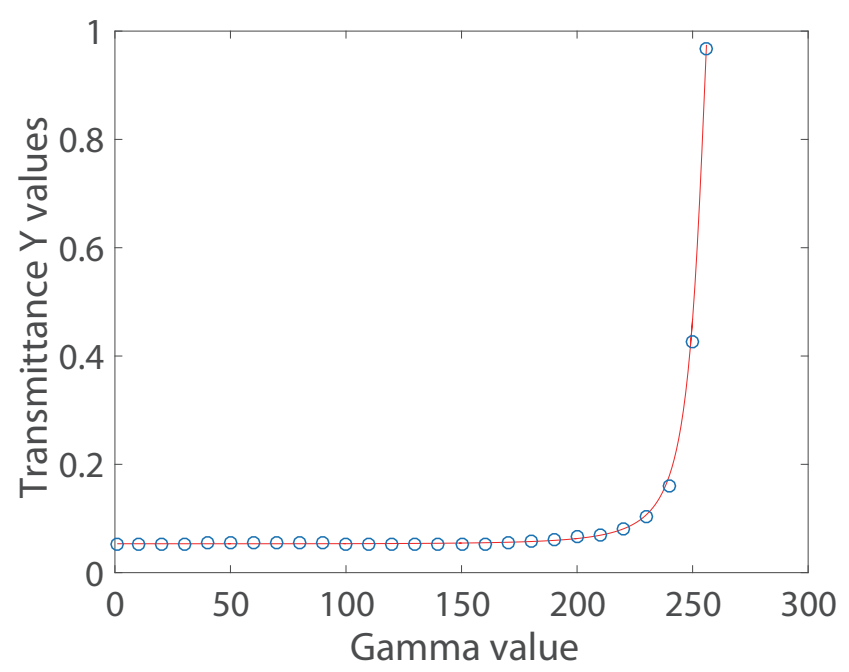

(a)

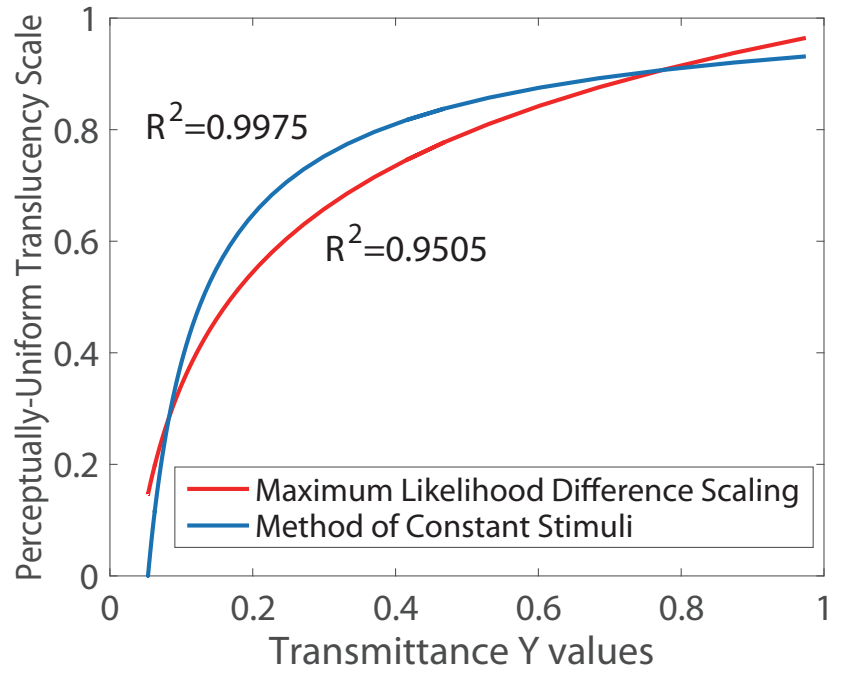

(b)

Figure 10. Gamma values vs. transmittance (a), power functions fitted to the relationship between transmittance and the perceptually-uniform translucency scale for both psychophysical methods (b).

\section{Psychometric Function}

The functions shown in Figure 9 depend on the printing setup and are different if, for instance, other material is employed or if the distribution of clear material in the samples is altered. A device-independent psychometric function $p$ can be obtained by measuring a physically specified translucency index of the samples and by mapping these measurements to the perceptually uniform translucency scale as follows:

$$
p=g \circ m^{-1}
$$

where $g$ is a function relating gamma values to the perceptuallyuniform scale (see Figure 9) and $m$ is an invertible function relating gamma values to measurements (see Figure 10(a)).

The remaining problem is to specify a measurement setup and conditions for obtaining a physically defined translucency in- dex. As explained in the introduction, light transport is a multidimensional phenomenon (spatially, directionally, spectrally as described by the BSSRDF). Reducing it to a single quantity results always in a major loss of information, i.e. there is in general a large set of materials with non-similar BSSRDFs possessing the same translucency index. This is analogously to metamerism in color science where spectral stimuli are mapped to trichromatic values.

In this paper, we define the translucency index to be the transmittance for a defined thickness (in this study $4 \mathrm{~mm}$ ) of the material and disregard any lateral light transport. This is of course a major simplification and we are working on developing a translucency index relying on both transmittance and later light transport. 
We measured the spectral transmittance of the samples using a Barbieri Spectro LFP transmission/reflectance spectrophotometer and computed the CIEXYZ $Y$ values that we normalized to one for a fully transparent sample. Figure 10(a) shows the transmittance and Figure 10(b) shows the psychometric functions. These functions were obtained by fitting a power function

$$
P_{T}(Y)=a Y^{b}+c
$$

to the data deduced from both experiments according to eq. (5). For the Method of Constant Stimuli, we obtain $(a, b, c)=(-$ $0.1367,-0.7022,1.071)$ and for the Maximum Likelihood Difference Scaling method $(a, b, c)=(-2.809,-0.08779,3.779)$. High $R^{2}$ values indicate that a power function accurately describes the relationship between transmittance and the perceptually uniform translucency scale.

\section{Conclusion and Future Work}

As expected, the psychometric curves obtained from both methods show the same trend. Due to the thickness and design of the samples and because only transmittance measurements are used, the psychometric function covers only the optically thin region of the sample set. Note that the transmittance is very small and almost constant in the gamma range from 0-180. In the remaining gamma range the relation between transmittance and perceptually uniform scale is well described by Stevens' power law [16].

It is noteworthy that lateral light transport increases also notably in the gamma range of $0-128$ as indicated by a significantly increased spread of an incident laser beam (not shown). However, the sensitivity to lateral light transport compared to transmittance is rather small, at least for the texture-less samples used in the experiments, as shown in Figure 2. This might be different if a texture is added and blurred by lateral light transport.

Even though both methods show the same trends and both experimental results can be well modeled by Stevens' power law, the resulting exponents - b in eq.(4) - are reasonably different. From literature we know [17] that as long as the experimental situation is kept reasonably constant, and the same measures of physical stimulus intensity are used, the average exponents produced by different groups of observers for the same continuum are quite similar. Since the experimental conditions were kept similar for both methods, the reason for deviations in the experimental results must be caused by the methods themselves.

In future work, we aim to also consider lateral light transport in our measurements - e.g. using a setup as proposed in [18]. For the psychophysics, we plan to investigate the impact of color texture on the observer judgements of lateral light transport.

\section{Acknowledgments}

This work was supported by the Leading Graduate School Program R03 of MEXT, by the FhG Internal Programs under Grant No. Attract 008-600075, by the Scan4Reco project funded by EU Horizon 2020 Framework Programme under grant agreement no 665091 and by JSPS KAKENHI Grant Number 15H05922 (Grant-in-Aid for Scientific Research on Innovative Areas "Innovative SHITSUKSAN Science and Technology" ).

\section{References}

[1] STRATASYS, Objet500 Connex3, http://www.stratasys.com/3dprinters/, 2014.

[2] 3D SYSTEMS, Projet 860Pro, http://www.3dsystems.com/3d-printers/professional/projet-860pro, 2014.

[3] MCOR TECHNOLOGIES, MCor Iris, http://mcortechnologies.com/3d-printers/iris/, 2014.

[4] M. Hašan, M. Fuchs, W. Matusik, H. Pfister and S. Rusinkiewicz, "Physical reproduction of materials with specified subsurface scattering," ACM Transactions on Graphics, vol. 29, no. 3, pp. 61, 2010.

[5] K. Vidimče, S.P. Wang, J. Ragan-Kelley and W. Matusik, "Openfab: A programmable pipeline for multi-material fabrication," ACM Transactions on Graphics, vol. 32, no. 4, pp. 136, 2013.

[6] "Cuttlefish," Available: https://www.cuttlefish.de/.

[7] A. Brunton, C.Arikan, P. Urban, "Pushing the limits of 3D color printing: error diffusion with translucent materials," ACM Transactions on Graphics, vol. 35, no. 1, pp. 4, 2015.

[8] F.E. Nicodemus, J.C. Richmond, J.J. Hsia, I.W. Ginsberg and T. Limperis, "Geometric considerations and nomenclature for reflectance," Monograph 160, National Bureau of Standards (US), 1977.

[9] R.S. Berns, D.H. Alman, L. Reniff, G.D. Snyder, and M.R. BalononRosen, "Visual determination of suprathreshold color-difference tolerances using probit analysis," Color Research and Application, vol. 16, pp. 297-316, 1991.

[10] L.T. Maloney and J.N. Yang, "Maximum likelihood difference scaling," Journal of Vision, vol. 3, no. 8, pp. 5-5, 2003.

[11] K. Knoblauch, L.T. Maloney, "Maximum likelihood difference scaling in R,” Journal of Statistical Software, vol. 25, no. 2, pp. 1-26, 2008.

[12] S. Nakauchi, R. Nishijima, T. Nagai, Y. Tani, K. Koida and M. Kitazaki, "Perceptual matching of translucent materials under different illuminant conditions," Journal of Vision, vol. 12, no. 9, pp. 867-867, 2011.

[13] P. Urban, M. Fedutina, and I. Lissner, "Analyzing small suprathreshold differences of LCD-generated colors," Journal of the Optical Society of America A, vol. 28, no. 7, pp. 1500-1512, 2011.

[14] MATLAB, R2015b and Curve Fitting Toolbox, Natick, Massachusetts: The MathWorks Inc., 2015.

[15] N. Prins and F.A.A. Kingdom, "Palamedes: Matlab routines for analyzing psychophysical data,". Available: www.palamedestoolbox.org.

[16] S. S.Stevens, "The psychophysics of sensory function," American scientist, vol. 48, no. 2, pp. 226-253, 1960.

[17] A. K. Myers, "Psychophysical scaling and scales of physical stimulus measurement," Psychological bulletin, vol. 92, no. 1, pp. 203, 1982.

[18] K. Happel, E. Dörsam and P. Urban, "Measuring Isotropic Subsurface Light Transport," Optics Express, vol. 22, no. 8, pp. 9048-9062, 2014.

\section{Author Biography}

Bui Minh Vu received his BE in Computer Science and Engineering from the Toyohashi University of Technology (2016), and now he is a master course student of Toyohashi University of Technology. He had worked as an internship student in the Fraunhofer Institute for Computer Graphics Research IGD in Darmstadt, Germany for 2 months. His work has focused on the visual perception and hyperspectral imaging. 